\section{PET/MRI Versus PET/CT for Whole-Body Staging}

TO THE EDITOR: The study by Martin et al. (1) comparing PET/MRI and PET/CT suffers from several methodologic concerns. Most importantly, all patients underwent PET/CT first and PET/MRI next. It is known that many malignant lesions will continue to increase target-to-background ${ }^{18}$ F-FDG uptake with delayed imaging (2,3). This methodologic flaw could have been mitigated by randomly alternating the order of PET/CT and PET/ MRI. The absence of such randomization biases the outcome of this comparison in favor of higher sensitivity for PET/MRI. The authors did not comment as to how many of the additional 155 lesions identified by PET/MRI were due to improved conspicuity in the PET images, as shown in Figures 5B and 5E, versus improved soft-tissue characteristics, as seen in Figure 3. The case in Figure 3 was from a patient with prostate cancer, for which ${ }^{18} \mathrm{~F}-\mathrm{FDG}$ PET is known to be less sensitive (4) and for which prostate-specific membrane antigen-labeled PET tracers will afford improved sensitivity versus ${ }^{18} \mathrm{~F}$-FDG (5). Next, why were the 2,686 non-wholebody PET/MRI studies, representing 2.6 times more subjects than the whole-body studies, excluded from comparison? Were these also due to "technical challenges" with the MRI exam? Next, of the 29 lesions (2.9\% of the total) found only by PET/MRI that were associated with a correction in the TNM stage, how many could have been expected to significantly alter the patient's treatment outcome had the initial PET/CT results been relied on? The authors have stated that the use of PET/MRI reduced the average radiation dose by $36 \%(3.9 \pm 1.3 \mathrm{mSv})$ compared with PET/CT scans using lowdose $\mathrm{CT}$ technique. If minimizing radiation dosimetry were a priority advocated by the authors, it is unclear why more than $80 \%$ of patients in the study underwent full-dose and not low-dose CT scans. The question could be asked as to the expected outcome benefit associated with an average 3.9-mSv reduction in absorbed dose by using PET/MRI in the targeted population of cancer patients, let alone in any patients (6). Finally, it is unlikely that a favorable cost benefit could be justified for more widespread use of PET/MRI in lieu of $\mathrm{PET} / \mathrm{CT}$, based on the small incremental improvement in lesions detected by PET/MRI as reported in this study.

\section{REFERENCES}

1. Martin O, Schaarschmidt BM, Kirchner J, et al. PET/MRI versus PET/CT for whole-body staging: results from a single-center observational study on 1,003 sequential examinations. J Nucl Med. 2020;61:1131-1136.

2. Chan WL, Ramsay SC, Szeto ER, et al. Dual-time-point ${ }^{18}$ F-FDG-PET/CT imaging in the assessment of suspected malignancy. J Med Imaging Radiat Oncol. 2011;55:379-390.

3. Kumar R, Loving VA, Chauhan A, et al. Potential of dual-time-point imaging to improve breast cancer diagnosis with ${ }^{18}$ F-FDG PET. $J$ Nucl Med. 2005;46:1819-1824.

4. Jadvar H. Is there utility for FDG PET in prostate cancer? Semin Nucl Med. 2016;46:502-506.

5. Maurer T, Eiber M, Schwaiger M, Gschwend JE. Current use of PSMA-PET in prostate cancer management. Nat Rev Urol. 2016;13:226-235.

6. Siegel JA, Pennington CW, Sacks B. Subjecting radiologic imaging to the linear no-threshold hypothesis: a non sequitur of non-trivial proportion. J Nucl Med. 2017;58:1-6.

COPYRIGHT @ 2021 by the Society of Nuclear Medicine and Molecular Imaging.

\author{
Stephen K. Gerard \\ Seton Medical Center \\ 1900 Sullivan Ave. \\ Daly City, CA 94015 \\ E-mail:skgnuc@comcast.net
}

Published online Oct. 9, 2020.

DOI: $10.2967 /$ jnumed.120.256453

\section{Radioguided Surgery}

TO THE EDITOR: Within the framework of a valuable initiative, the supplement to the December issue of The Journal of Nuclear Medicine highlights some of the major contributions that nuclear medicine and molecular imaging have made to patient care over 60 years of publication. An additional area that deserves mention is radioguided surgery, which starts with the nuclear medicine procedure of tagging with a radioactive label (administered either systemically or locoregionally) a certain tissue or lesion to ease its identification by preoperative imaging and its subsequent resection by an intraoperative counting probe. There have been several thousand citations to articles published in The Journal of Nuclear Medicine regarding radioguided sentinel lymph node (SLN) biopsy, contributing to the recognition of this procedure as the standard of care for some cancers. After Cabañas introduced in the 1970s the SLN concept as an anatomic notion (1), in 1992 Morton renewed interest in the SLN approach using visual guidance with a blue dye to visualize lymphatic drainage from tumors, recognizing its variability from patient to patient (2). Nonetheless, it was the introduction of radioguidance in the mid1990s that led to the current array of clinical applications of SLN surgery - as witnessed by the number of publications in this field, which have increased by more than 10-fold every 5 years between 1996 and 2005 versus 1991-1995.

As a fundamental aid for primary staging of solid epithelial cancers, radioguided SLN biopsy constitutes one of the best examples of how nuclear medicine interacts with and has a crucial impact on other medical specialties. In fact, this procedure constitutes the undisputed standard of care for initial treatment of cutaneous melanoma and breast cancer, and it is increasingly being recognized as the standard of care also for penile cancer, head and neck cancers, and some gynecologic cancers. Radioguided surgery, including robot-assisted procedures, is undergoing clinical validation in other malignancies, not only for SLN biopsy but also for radiotagged tumor resection.

Ranking of articles published in The Journal of Nuclear Medicine according to the number of citations in the international literature identifies the top 5 articles as milestone contributions to establish radioguided SLN biopsy as the standard of care, particularly for breast cancer and cutaneous melanoma (3-7). The next 5 most cited articles (8-12) deal with important components of radioguided SLN surgery that ensure optimal performance of the procedure, as well as technologic advances based on fruitful interactions of nuclear medicine with other medical specialties. In particular, they emphasize the crucial role of preoperative imaging within the whole procedure of 